\title{
Solving Bernoulli Differential Equations by using Newton's Interpolation and Aitken's Methods
}

\author{
Nasr Al Din IDE* \\ Aleppo University-Faculty of Science-Department of Mathematics
}

*Corresponding Author: Nasr Al Din IDE, Aleppo University-Faculty of Science-Department of Mathematics

\begin{abstract}
One of important problem in Mathematics is solving differential equations by analytic methods and numerical methods. Most of researchers treated numerical methods to solve first order ordinary differential equations. These methods such as Taylor series method, Runge Kutta method and Euler's method, etc. Faith Chelimo Kosgei studied this problem by combined the newton's interpolation and Lagrange method, Nasr Al Din IDE also studied this problem by Using Newton's Interpolation and Aitken's Method for Solving Riccati First Order Differential equation. This study will use Newton's interpolation and Aitken's method to solve Bernoulli Differential Equations, some examples treated to illustrate the efficiency of this method.
\end{abstract}

Keywords: Differential equation, Bernoulli Differential Equations, Analytic method, Numerical method, newton's interpolation method, Aitken Methods

\section{INTRODUCTION}

In Mathematics many of problems can be formulated to form the ordinary differential equation, specially Bernoulli differential equations of first order, here we study and solve the Bernoulli differential equations. A numerical method is used to solve numerical problems. The differential equation problem [1-10], consists of at least one differential equation and at least one additional equation such that the system together have one and only one solution called the analytic or exact solution to distinguish it from the approximate numerical solutions that we shall consider in this paper of first order, Faith C. K [1] studied the problem of Riccati by using combination of newton's interpolation and Lagrange method, Nasr Al Din Ide [2,3] studied this problem also by using of Newton's Interpolation and Lagrange Method for Solving Bernolli equation and he combined of Newton's interpolation and Aitken's method as hybrid technique by using these two types of interpolation to solve first order differential equation. In present study we will study Bernoulli Differential Equations by combined of Newton's interpolation and Aitken's method [4-10]. Finally we verified on a number of examples and numerical results obtained show the efficiency of the method given by present study in comparison with the exact solution.

Let the Bernoulli differential equation which can be written in the following standard form:

$y^{\prime}+\mathrm{P}(\mathrm{x}) \mathrm{y}=\mathrm{Q}(\mathrm{x}) y^{n}$

where $\mathrm{P}$ and $\mathrm{Q}$ are functions of $\mathrm{x}$, and $\mathrm{n}$ is a constant

$\mathrm{n} \neq 1$ (the equation is thus nonlinear).

Where y is a known function and the values in the initial conditions are also known numbers.

\section{Present Aitken Interpolation Method}

\subsection{Combined Newton's Interpolation and Lagrange Method [1, 2]}

This study combine both Newton's interpolation method and Lagrange method. it used newton's interpolation method to find the second two terms then use the three values for $y$ to form a quadratic equation using Lagrange interpolation method as follows;

\subsubsection{Newton's interpolation method [1, 2, 9]}

$f_{n}(x)=a_{0}+a_{1}\left(x-x_{0}\right)+a_{2}\left(x-x_{0}\right)\left(x-x_{1}\right)+\ldots+a_{n}\left(x-x_{0}\right)\left(x-x_{1}\right) \ldots a_{2}\left(x-x_{n-1}\right)$

Where 
$a_{0}=y_{0}, \quad a_{1}=\frac{f\left(x_{1}\right)-f\left(x_{0}\right)}{\left(x_{1}-x_{0}\right)}, \quad a_{2}=\frac{\frac{f\left(x_{2}\right)-f\left(x_{1}\right)}{\left(x_{2}-x_{1}\right)}-\frac{f\left(x_{1}\right)-f\left(x_{0}\right)}{\left(x_{1}-x_{0}\right)}}{\left(x_{2}-x_{0}\right)}$

Etc

\subsubsection{Lagrang interpolation method $[1,8]$}

$y_{n}=\frac{\left(x-x_{1}\right)-\left(x-x_{2}\right)}{\left(x_{0}-x_{1}\right)\left(x_{0}-x_{2}\right)} y_{0}+\frac{\left(x-x_{0}\right)-\left(x-x_{2}\right)}{\left(x_{1}-x_{0}\right)\left(x_{1}-x_{2}\right)} y_{1}+\frac{\left(x-x_{0}\right)-\left(x-x_{1}\right)}{\left(x_{2}-x_{0}\right)\left(x_{2}-x_{1}\right)} y_{2}$

\section{DESCRIPTION OF THE METHOD}

This method will combine both Newton's interpolation method and Lagrange method .it used newton's interpolation method to find the second two terms then use the three values for y to form a linear or quadratic equations using Lagrange interpolation method as follows;

$f_{n}(x)=a_{0}+a_{1}\left(x-x_{0}\right)+a_{2}\left(x-x_{0}\right)\left(x-x_{1}\right)+\ldots+a_{n}\left(x-x_{0}\right)\left(x-x_{1}\right) . . a_{2}\left(x-x_{n-1}\right)$

Where

$a_{0}=y_{0}, a_{1}=\frac{f\left(x_{1}\right)-f\left(x_{0}\right)}{\left(x_{1}-x_{0}\right)}, \quad \frac{\frac{f\left(x_{2}\right)-f\left(x_{1}\right)}{\left(x_{2}-x_{1}\right)}-\frac{f\left(x_{1}\right)-f\left(x_{0}\right)}{\left(x_{1}-x_{0}\right)}}{\left(x_{2}-x_{0}\right)}$

etc

$y_{1}=a_{0}+a_{1}\left(x-x_{0}\right)$

$y_{2}=a_{0}+a_{1}\left(x-x_{0}\right)+a_{2}\left(x-x_{0}\right)\left(x-x_{1}\right)$

Forming quadratic interpolation of Lagrange, we have:

$y_{n}=\frac{\left(x-x_{1}\right)\left(x-x_{2}\right)}{\left(x_{0}-x_{1}\right)\left(x_{0}-x_{2}\right)} \cdot y_{0}+\frac{\left(x-x_{0}\right)\left(x-x_{2}\right)}{\left(x_{1}-x_{0}\right)\left(x_{1}-x_{2}\right)} \cdot y_{1}+\frac{\left(x-x_{0}\right)\left(x-x_{1}\right)}{\left(x_{2}-x_{0}\right)\left(x_{2}-x_{1}\right)} \cdot y_{2}$

Note: we can use Newton's Forward Interpolation Formula instead of Newton's divided Interpolation method in (2.1).

\subsection{Aitken interpolation method $[3,8]$}

$$
\begin{aligned}
& \mathrm{P}_{\mathrm{o}, \mathrm{k}}(\mathrm{x})=\frac{1}{\mathrm{x}_{\mathrm{k}}-\mathrm{x}_{\mathrm{o}}}\left|\begin{array}{ll}
\mathrm{y}_{\mathrm{o}} & \mathrm{x}_{\mathrm{o}}-\mathrm{x} \\
\mathrm{y}_{\mathrm{k}} & \mathrm{x}_{\mathrm{k}}-\mathrm{x}
\end{array}\right| \\
& \mathrm{P}_{\mathrm{o}, 1,2}(\mathrm{x})=\frac{1}{\mathrm{x}_{2}-\mathrm{x}_{1}}\left|\begin{array}{ll}
\mathrm{P}_{\mathrm{o}, 1}(\mathrm{x}) & \mathrm{x}_{1}-\mathrm{x} \\
\mathrm{P}_{\mathrm{o}, 2}(\mathrm{x}) & \mathrm{x}_{2}-\mathrm{x}
\end{array}\right| \\
& y_{n}=P_{o, 1,2, \ldots, n}(x)=\frac{1}{x_{n}-x_{n-1}}\left|\begin{array}{cc}
P_{o, 1, \ldots,(n-1)}(x) & x_{n-1}-x \\
P_{o, 1, \ldots,(n-2), n}(x) & x_{n}-x
\end{array}\right|
\end{aligned}
$$

\section{EXAMPLES}

In this section, we will check the effectiveness of the present technique (3). First numerical comparison for the following test examples taken in [3].

\section{Example 1}

Solve $y^{\prime}=y+x \cdot \mathrm{y}^{\frac{1}{2}}$, the exact solution of this problem is

$y=\left(c \cdot \mathrm{e}^{\frac{x}{2}}-x-2\right)^{2}$

For $\mathrm{c}=1$, the exact solution of this problem is $y=\left(\mathrm{e}^{\frac{x}{2}}-x-2\right)^{2}$, hence, $y(0)=1$

Now, by taking the step $\mathrm{h}=0.01$

First by using Newton's interpolation, we have

$a_{0}=1=y_{0}$

$a_{1}=\frac{f\left(x_{1}\right)-f\left(x_{0}\right)}{\left(x_{1}-x_{0}\right)}=\left[\frac{d y}{d x}\right]_{0,1}=0$

$y_{1}=1+0(0.01-0)=1$

$a_{2}=\frac{\frac{f\left(x_{2}\right)-f\left(x_{1}\right)}{\left(x_{2}-x_{1}\right)}-\frac{f\left(x_{1}\right)-f\left(x_{0}\right)}{\left(x_{1}-x_{0}\right)}}{\left(x_{2}-x_{0}\right)}=\frac{\left[\frac{d y}{d x}\right]_{0.010 .01}-\left[\frac{d y}{d x}\right]_{0,0}}{0.02-0}=0.55$

$y_{2}=1+0(0.02-0)+0.55(0.02-0)(0.02-0.01)=1.000110000$

Now, forming linear and quadratic using Aitken Method 


$$
\begin{aligned}
& P_{0,1}(x)=1 \\
& P_{0,2}(x)=0.0055 x+1 \\
& P_{0,1,2}(x)=0.55 x^{2}-0.0055 x+1
\end{aligned}
$$

Hence, we can take the approximation solution of linear and quadratic using Aitken Method, if we take quadratic using Aitken Method, Table 1 gives the approximation solutions of Runge-Kutta method and Combined Newton's Interpolation and Aitken method with the exact solution of example 1 with the errors for :

$\mathrm{x}=0,0.01,0.02,0.03,0.04,0.05,0.06,0.07,0.08,0.09,0.1$

Table1. Solution of $\quad y^{\prime}=y+x \cdot y^{\frac{1}{2}}, \quad y(0)=1$

\begin{tabular}{|c|c|c|c|}
\hline $\mathbf{x}$ & $\begin{array}{c}\text { Combined Newton's } \\
\text { Interpolation and Aitken }\end{array}$ & \multicolumn{1}{|c|}{ exact Values } & Absolut error \\
\hline 0 & 1 & 1 & 0 \\
\hline 0.01 & 1 & 1.009999833 & 0.009999833 \\
\hline 0.02 & 1.000110000 & 1.019998665 & 0.019888665 \\
\hline 0.03 & 1.000330000 & 1.029995492 & 0.029665492 \\
\hline 0.04 & 1.000660000 & 1.039989307 & 0.039329307 \\
\hline 0.05 & 1.001100000 & 1.049979102 & 0.048879102 \\
\hline 0.06 & 1.001650000 & 1.059963867 & 0.058313867 \\
\hline 0.07 & 1.002310000 & 1.069942587 & 0.067632587 \\
\hline 0.08 & 1.003080000 & 1.089877829 & 0.076834247 \\
\hline 0.09 & 1.003960000 & 1.0900000000 & 0.086040000 \\
\hline 0.1 & 1.004950000 & 1.100000000 & 0.095050000 \\
\hline
\end{tabular}

\section{Example 2}

Solve $y^{\prime}=2 x y+2 x^{3} \cdot y^{2}$, the exact solution of this problem is

$y=1 /\left(c \cdot \mathrm{e}^{-\mathrm{x}^{2}}+1-\mathrm{x}^{2}\right)$

For $\mathrm{c}=0$, the exact solution of this problem is $y=1 /\left(1-\mathrm{x}^{2}\right)$, hence, $y(0)=1$

Now, by taking the step $\mathrm{h}=0.01$

First by using Newton's interpolation, we have

$$
\begin{aligned}
& a_{0}=1=y_{0} \\
& a_{1}=\frac{f\left(x_{1}\right)-f\left(x_{0}\right)}{\left(x_{1}-x_{0}\right)}=\left[\frac{d y}{d x}\right]_{0,1}=0 \\
& y_{1}=1+0(0.01-0)=1 \\
& a_{2}=\frac{\frac{f\left(x_{2}\right)-\left(x_{1}\right)}{\left(x_{2}-x_{1}\right)}-\frac{f\left(x_{1}\right)-f\left(x_{0}\right)}{\left(x_{1}-x_{0}\right)}}{\left(x_{2}-x_{0}\right)}=0.01000001 \\
& y_{2}=1.000002
\end{aligned}
$$

Now, forming linear and quadratic using Aitken Method

$P_{0,1}(x)=1$

$P_{0,2}(x)=0.0001 x+1$

$P_{0,1,2}(x)=0.01 x^{2}-0.0001 x+1$

Hence, we can take the approximation solution of linear and quadratic using Aitken Method, if we take quadratic using Aitken Method, Table 2 gives the approximation solution and the exact solution of example 1 with the error for : $\mathrm{x}=0,0.01,0.02,0.03,0.04,0.05,0.06,0.07,0.08,0.09,0.1$.

Table2. Solution of $y^{\prime}=2 x y+2 x^{3} \cdot y^{2}, \quad y(0)=1$

\begin{tabular}{|c|c|c|c|}
\hline $\mathbf{x}$ & $\begin{array}{c}\text { Combined Newton's } \\
\text { Interpolation and Aitken }\end{array}$ & exact Values & Absolut error \\
\hline 0 & 1 & 1 & 0 \\
\hline 0.01 & 1.000009000 & 1.000100010 & 0.000099110 \\
\hline 0.02 & 1.000002000 & 1.000400610 & 0.000381600 \\
\hline
\end{tabular}


Solving Bernoulli Differential Equations by using Newton's Interpolation and Aitken's Methods

\begin{tabular}{|l|l|l|l|}
\hline \multicolumn{3}{|l|}{} \\
\hline 0.03 & 1.000006000 & 1.000900811 & 0.000394160 \\
\hline 0.04 & 1.000012000 & 1.001602564 & 0.001590564 \\
\hline 0.05 & 1.002495000 & 1.002506266 & 0.002566760 \\
\hline 0.06 & 1.000093000 & 1.003613007 & 0.003583007 \\
\hline 0.07 & 1.000042000 & 1.004924128 & 0.004882128 \\
\hline 0.08 & 1.000056000 & 1.006441224 & 0.006385224 \\
\hline 0.09 & 1.000072000 & 1.008166146 & 0.008094146 \\
\hline
\end{tabular}

\section{Example 3}

Solve $y^{\prime}=\mathrm{x}^{3} \cdot \mathrm{y}^{3}-x y$, the exact solution of this problem is

$y=1 /\left(c \cdot \mathrm{e}^{\mathrm{x}^{2}}+1+\mathrm{x}^{2}\right)$

For $\mathrm{c}=0$, the exact solution of this problem is $y=1 /\left(1+\mathrm{x}^{2}\right)$, hence, $y(0)=1$

Now, by taking the step $\mathrm{h}=0.01$

First by using Newton's interpolation, we have

$a_{0}=1=y_{0}$

$a_{1}=\frac{f\left(x_{1}\right)-f\left(x_{0}\right)}{\left(x_{1}-x_{0}\right)}=\left[\frac{d y}{d x}\right]_{0,1}=0$

$y_{1}=1+0(0.01-0)=1$

$\underline{f\left(x_{2}\right)-\left(x_{1}\right)}-\underline{f\left(x_{1}\right)-f\left(x_{0}\right)}$

$a_{2}=\frac{\left(x_{2}-x_{1}\right)}{\left(x_{2}-x_{0}\right)}=-0.005$

$y_{2}=0.999999$

Now, forming linear and quadratic using Aitken Method

$P_{0,1}(x)=1$

$P_{0,2}(x)=-0.00005 x+1$

$P_{0,1,2}(x)=-0.005 x^{2}+0.00005 x+1$

Hence, we can take the approximation solution of linear and quadratic using Aitken Method, if we take quadratic using Aitken Method, Table 3 gives the approximation solution and the exact solution of example 1 with the error for :

$\mathrm{x}=0,0.01,0.02,0.03,0.04,0.05,0.06,0.07,0.08,0.09,0.1$.

Table3. Solution of $y^{\prime}=x^{3} \cdot y^{3}-x y, \quad y(0)=1$

\begin{tabular}{|c|c|c|c|}
\hline $\mathbf{x}$ & $\begin{array}{c}\text { Combined Newton's } \\
\text { Interpolation and Aitken }\end{array}$ & \multicolumn{1}{|c|}{ exact Values } & \multicolumn{1}{c|}{ Absolut error } \\
\hline 0 & 1 & 1 & \multicolumn{1}{|c|}{0} \\
\hline 0.01 & 1 & 0.999900010 & 0.000099990 \\
\hline 0.02 & 0.999999000 & 0.999600160 & 0.000398840 \\
\hline 0.03 & 0.999997000 & 0.999100809 & 0.000896191 \\
\hline 0.04 & 0.999994000 & 0.998402556 & 0.001591444 \\
\hline 0.05 & 0.999990000 & 0.997506234 & 0.002483766 \\
\hline 0.06 & 0.999985000 & 0.996412914 & 0.003572086 \\
\hline 0.07 & 0.999979000 & 0.995123893 & 0.004855107 \\
\hline 0.08 & 0.999972000 & 0.993640700 & 0.004855107 \\
\hline 0.09 & 0.999964000 & 0.991965083 & 0.007998917 \\
\hline 0.1 & 0.999950500 & 0.990099010 & 0.009851490 \\
\hline
\end{tabular}

\section{CONCLUSION}

In this paper, we have been applied the combined Newton's interpolation and Aitken method to solve nonlinear Bernoulli differential equation of first order, we find a good result compared to the exact solution through a three examples showing that. 


\section{REFERENCES}

[1] Faith C. K, SOLUTION OF FIRST ORDER DIFFERENTIAL EQUATION USING NUMERICAL NEWTON'S INTERPOLATION AND LAGRANGE METHOD, International Journal of Development Research, ISSN: 2230-9926, Vol. 08, Issue, 02, pp.18973-18976, February, 2018

[2] Nasr Al Din IDE, Solving Bernolli Differential Equations by Using Newton's Interpolation and Lagrange Method, Computational and Applied Mathematical Sciences, Idosi Publications, 5 (1): 18-22, 2020.

[3] Nasr Al Din IDE, Using Newton's Interpolation and Aitken's Method for Solving First Order Differential Equation, World Applied Sciences Journal, Idosi Publications, 38 (3): 191-194, 2020.

[4] Graham S McDonald, Differential Equations, BERNOULLI EQUATIONS, A Tutorial Module for learning how to solve Bernoulli differential equations, 2004, g.s.mcdonald@salford.ac.uk.

[5] S. Islam, Y. Khan, N. Faraz and F. Austin, Numerical solution of logistic differential equations by using the Laplace decomposition method, World Appl. Sci. J., 8, 1100-1105(2010).

[6] Isaacson E., Keller H.B., Analysis of numerical methods, Second edition, Dover, Mineola, NY, 1994.

[7] Stoer J., Burlisch R., Introduction to numerical analysis, Second edition, Springer-Verlag, New York, NY, 1993.

[8] Numerical Analysis, Richard L. Burden, J. Douglas Faires, 1989, PWS-KENT Publishing Company, Boston.

[9] Davis P.J., Interpolation and approximation, Second edition, Dover, New York, NY, 1975.

[10] " The Numerical Solution of ordinary and partial differential equations" GRANVILLE SEWELL1992, ACADEMIC PRESS, INC, New York.

Citation: Nasr Al Din IDE, Solving Bernoulli Differential Equations by using Newton's Interpolation and Aitken's Methods, International Journal of Scientific and Innovative Mathematical Research (IJSIMR), vol. 8, no. 6, pp. 1-5, 2020. Available : DOI: https://doi.org/10.20431/2347-3142.0806001

Copyright: (C) 2020 Authors. This is an open-access article distributed under the terms of the Creative Commons Attribution License, which permits unrestricted use, distribution, and reproduction in any medium, provided the original author and source are credited. 